\title{
Contribution a l'étude des effets de croisement sur la taille de la portée et la croissance des lapereaux : Cas de la ferme agro- pastorale Jacarandas en R.D.Congo
}

\author{
Museba, W1.; Kashala, K².; Mutondo, L³.; Lunumbi, 01.; Kumwimba, L4. \\ 1 Service d'Economie Animale et Gestion des Entreprises d'Elevage, Faculté de Médecine Vétérinaire, Université de \\ Lubumbashi, BP 1825, Lubumbashi, RD Congo. \\ 2 Service d'Amélioration Génétique, Faculté de Médecine Vétérinaire, Université de Lubumbashi, BP 1825, RD \\ Congo. \\ ${ }^{3}$ Chercheur indépendant, Lubumbashi, RD Congo. \\ ${ }^{4}$ Service Biochimie, Faculté de Médecine, Université de Kalemie, RD Congo. \\ Correspondent: kmusebawaanda@gmail.com
}

Original submitted in on 23rd March 2016. Published online at www.m.elewa.org on 31st August 2016 http://dx.doi.org/10.4314/jab.v104i1.4

\section{RESUME}

Objectif : était de déceler les produits plus performants (poids et taille) de lapins par de divers croisements en appliquant les méthodes d'amélioration génétique pouvant améliorer leur productivité afin d'assurer un avenir meilleur de l'élevage.

Méthodologie et résultats: Pour l'obtention des résultats, nous nous sommes servis d'une balance électronique, qui nous a permis d'effectuer les pesées selon le protocole établi, aux jours 15, 30, 45 et 150 avec un sevrage qui intervenait à 60 jours de la naissance. L'étude a porté sur un effectif de 20 lapins, dont 3 mâles de races Alaska, Néozélandaise et Chinchilla en croisements multiples avec 17 lapines de races Argenté de champagne, Californien, Chinchilla, Néozélandaise et Papillon. A l'issu de ces croisements ; 113 lapereaux ont été obtenus, parmi lesquels 17 lapereaux sont morts avant la date de la première pesée, qui n'ont pas été pris en compte lors de l'étude. II s'est ainsi dégagé une portée moyenne de $6,65 \pm 2,12$ lapereaux à la première portée de notre étude. Selon l'évolution pondérale des lapereaux; $0,300 \pm 0,11 \mathrm{~kg}$ a été la moyenne observée à la première pesée, sur l'ensemble des croisements. Cette évolution a été plus importante pour le produit issu du croisement de Néozélandais $\times$ Néozélandais $(0,621 \pm 0,12 \mathrm{~kg})$. A 30 jours d'âge, les lapereaux issus de Néozélandais $\times$ Néozélandais ont révélé une moyenne supérieure de $0,836 \pm 0,19 \mathrm{~kg}$, bien que la moyenne des croisements ait été $0,498 \pm 017 \mathrm{~kg} /$ /lapereau. II s'en est suivi une moyenne de 0,770 $\pm 0,27 \mathrm{~kg} /$ lapereau à 45 jours d'âge. A l'entrée en reproduction (150 jours), le même produit s'est démarqué des autres croisements avec $2,900 \pm 0,40 \mathrm{~kg}$ /lapin, bien que la moyenne pondérale pour cette catégorie d'âge soit 2,130 $\pm 0,45 \mathrm{~kg} / \mathrm{lapin}$.

Conclusion: L'utilisation des géniteurs à grande prolificité en croisement permet d'augmenter la productivité numérique des cheptels lapins. A cet effet, le mâle Alaska et Néo-Zélandais sont d'une grande utilité pour l'augmentation de l'effectif et leur utilisation devra être rationnelle.

Mots-clés : Étude, croissance, lapereaux, effet de la croissance,. 


\section{ABSTRACT}

Objective: To evaluate the best performing products (weight and height) of rabbits of various crosses applying genetic improvement methods that can improve productivity to ensure a better future breeding.

Methodology and results: For the obtaining of the results, we used an electronic balance, which allowed us to make weighing according to the established protocol, in the days 15, 30, 45 and 150 with a weaning at 60 days after the birth. The study concerned 20 rabbits, among which 3 purebred males Alaska, New Zealander and Chinchilla in multiple crossings with 17 purebred rabbits Silvered of champagne, Californian, Chinchilla, New Zealander and Butterfly. From these crosses; 113 young rabbits were obtained, among whom 17 young rabbits died before the date of the first weighing, which were not taken into account during the study. An average of $6.65 \pm 2.12$ young rabbits in the first obtained. According to the weight evolution of young rabbits; $0.300 \pm 0.11 \mathrm{~kg}$ was the average observed in the first weighing, on the set of the crossings. This evolution was more important for the product stemming from the crossing of New Zealander $\times$ New Zealand $(0.621 \pm 0.12 \mathrm{~kg})$. In 30 days of age, young rabbits stemming from New Zealander $\times$ New Zealand revealed an average superior of $0,836 \pm 0.19 \mathrm{~kg}$, although average of the crossings was $0,498 \pm 017$ $\mathrm{kg} /$ young rabbit. It followed an average of $0.770 \pm 0.27 \mathrm{~kg} / \mathrm{young}$ rabbit in 45 days of age. In the entrance to reproduction (150 days), the same product distanced itself from other crossings with $2.900 \pm 0.40$ $\mathrm{kg} / \mathrm{rabbit}$, although the weight average for this category of age is $2.130 \pm 0.45 \mathrm{~kg} / \mathrm{rabbit}$.

Conclusion: the use of the parents with big prolificacy in crossing allows increasing productivity of the rabbits. For that purpose, male Alaska and New Zealander are of a big utility for the increase of the colony and their use will have to be rational.

Keywords: Study, growth, young rabbits, effect of the growth,.

\section{INTRODUCTION}

Depuis 1960, la population humaine a globalement augmenté. Cette augmentation a été plus importante dans les pays en développement que ceux du monde industriel. II en est de même pour le bétail, où toutes les espèces ont vu leurs effectifs augmenter et dont l'accroissement a été plus perceptible pour les monogastriques (porc et volailles) que les polygastriques (FAO, 1998). En Afrique en général, la demande des consommateurs en protéines d'origine animale est de plus en plus élevée. Pour participer à la couverture de ces besoins, la mise sur pied des élevages à cycles courts s'avère indispensable. Ainsi l'élevage de petites espèces animales peut représenter une opération hautement lucrative aussi bien pour les petits fermiers que pour les exploitants sans terres. De toutes ces petites espèces, l'élevage du lapin est de loin le plus répandu et le mieux adapté au petit exploitant, particulièrement dans les régions méditerranéennes (Schiere, 2004). De surcroît, la cuniculture exige peu de tabous religieux ou autres reposant sur la chair de lapin (sauf dans les cultures végétariennes) et même l'islam n'en interdit pas la consommation (Combes et al.,2015). Le développement de cet élevage nécessite non seulement une sélection et un croisement adéquat des lapins, mais aussi des études approfondies dans certains domaines tels que la génétique, la pathologie et l'alimentation qui pourraient contribuer de ce fait, à la sécurisation alimentaire des éleveurs et à la santé humaine (Akoutey et Kpodekon 2009). Pour répondre à tous ces besoins, les croisements entre différentes races lapines élevées à Lubumbashi seraient une alternative, en vue d'atteindre les résultats de performance attendus par les éleveurs et de ces races croisées; sortirait un croisement qui donnerait satisfaction aux besoins de ces derniers et aux consommateurs surtout que la viande de lapins constitue une originale solution rechange au poulet par sa viande blanche, dont il rappelle un peu la saveur (Combes, 2004). Sa viande maigre offre une bonne source des protéines complètes et regorge en plus les acides aminés essentiels (Lysine Leucine Arginine); des vitamines et minéraux notamment vitamines B3, B6, B12, phosphore, et selenium (Combes, 2004). 
Museba et al . J. Appl. Biosci. 2016 Contribution a l'étude des effets de croisement sur la taille de la portée et la croissance des lapereaux : Cas de la ferme agro- pastorale Jacarandas en R.D.Congo

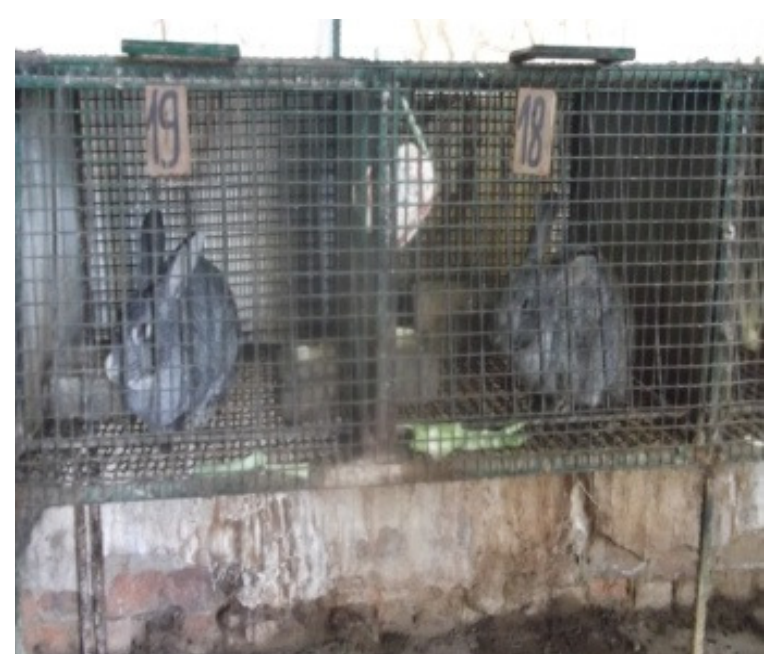

Deux femelles reproductrices en attente de saillie

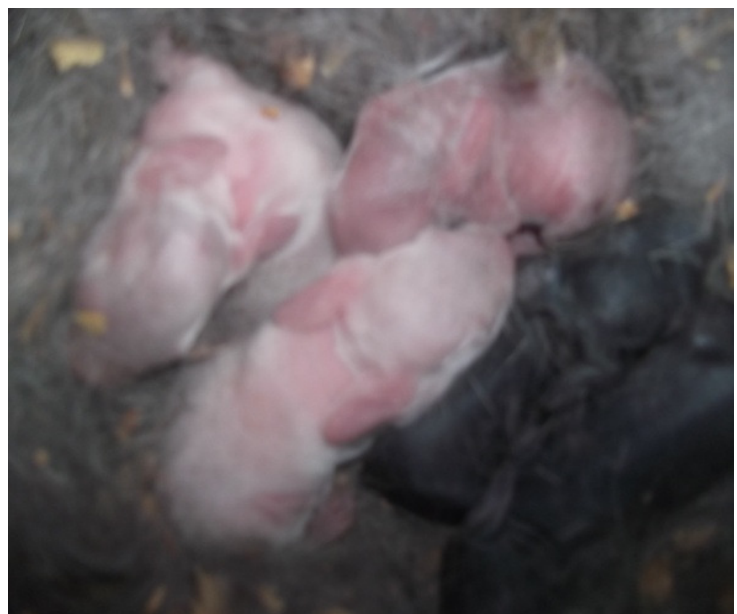

Lapereaux issus du croisement Néo zélandais - californiens

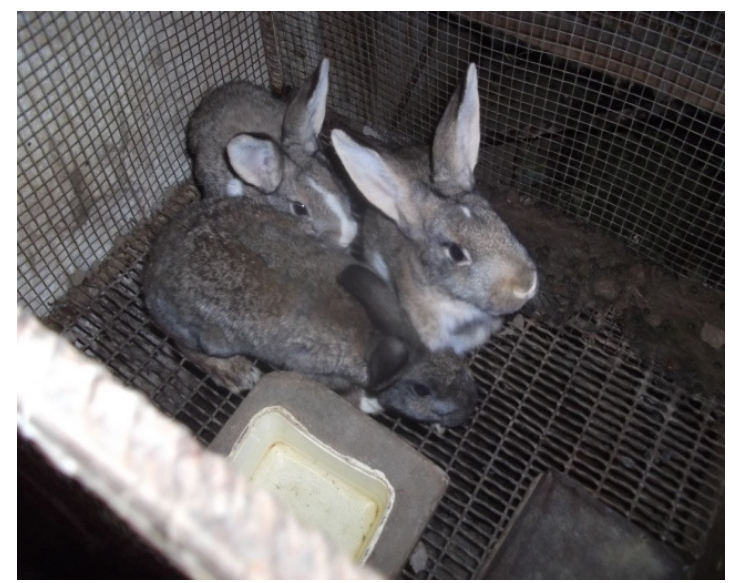

Lapereaux à l'engraissement

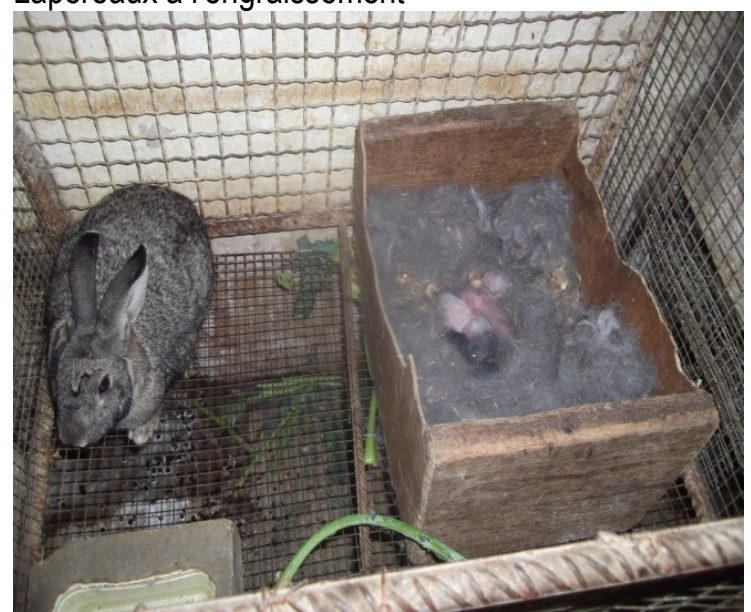

Lapine Chinchilla dans une cage avec sa portée

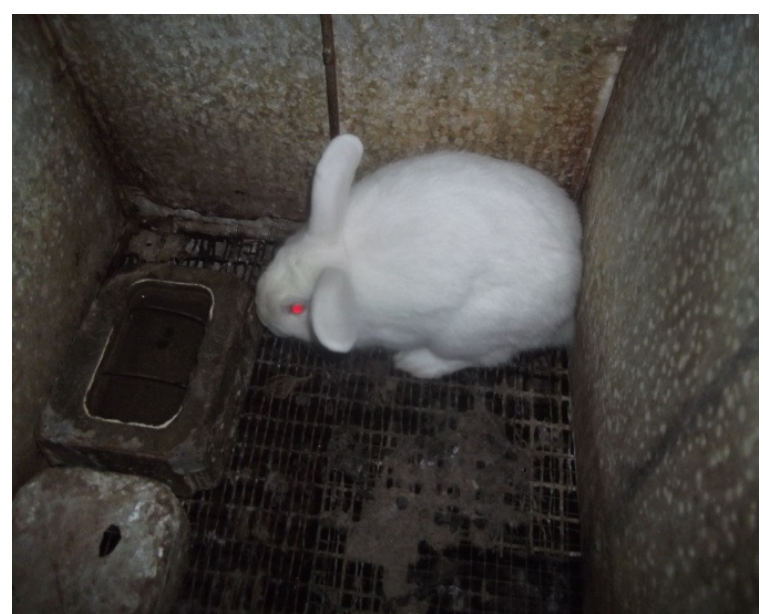

Lapin Néo-zélandais en cage 


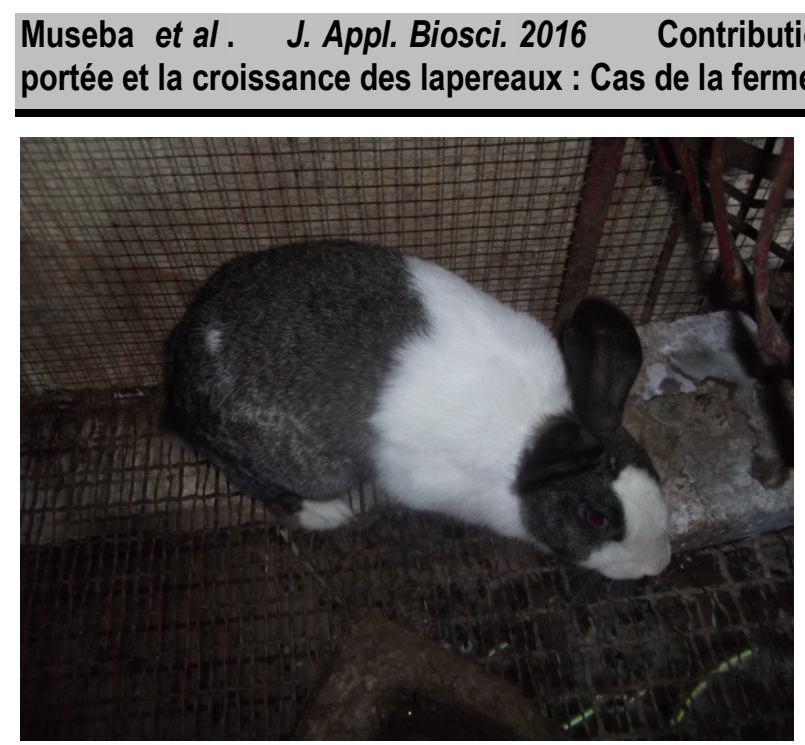

Lapin issu du croissement californien - néo zélandais

Cette viande blanche contient aussi des lipides, parmi lesquelles les acides gras saturés (acides palmitiques) qui se retrouvent nettement inférieurs à la quantité présente dans les viandes rouges et dont la consommation exagérée chez l'homme augmenterait des risques de cancer de l'œsophage (De Stefani et Deneo-Pellegrini 1999) et colorectal (Giovannucci et al., 1994; Riboli et Norat, 2001). Parmi les acides gras insaturés, les mono insaturés (acides oléiques) contenus dans la viande du lapin abaissent le taux de cholestérol total sans modifier la concentration en cholestérol HDL (piers et al., 2003) et réduiraient chez l'Homme les risques des maladies

\section{MATERIEL ET METHODES}

Cette étude a été menée à la ferme agro-pastorale JACARANDAS ; ferme à vocation laitière, touristique et professionnelle. Elle est située à $17 \mathrm{~km}$ du centre-ville de Lubumbashi sur la route Munama, dans la commune annexe, Province du Haut-Katanga, District de Lubumbashi, en République Démocratique du Congo. Cette ferme a une superficie de 152 hectares et exploite non seulement les Bovins de type Frison, mais également les porcins, la volaille, le cobaye et le lapin. Concernant le lapin ; aucun n'est à priori de race pure, en raison des multiples croisements déjà effectués pendant plusieurs années. II s'agit ici d'une question de prédominance des caractères raciaux. Concernant le climat ; il est du type tropical humide caractérisé par six mois de saison sèche, auxquels succèdent six mois pluvieux (Van, 1994). Pour ce qui est de la végétation ; elle est parsemée d'arbres et arbustes; mais aussi des

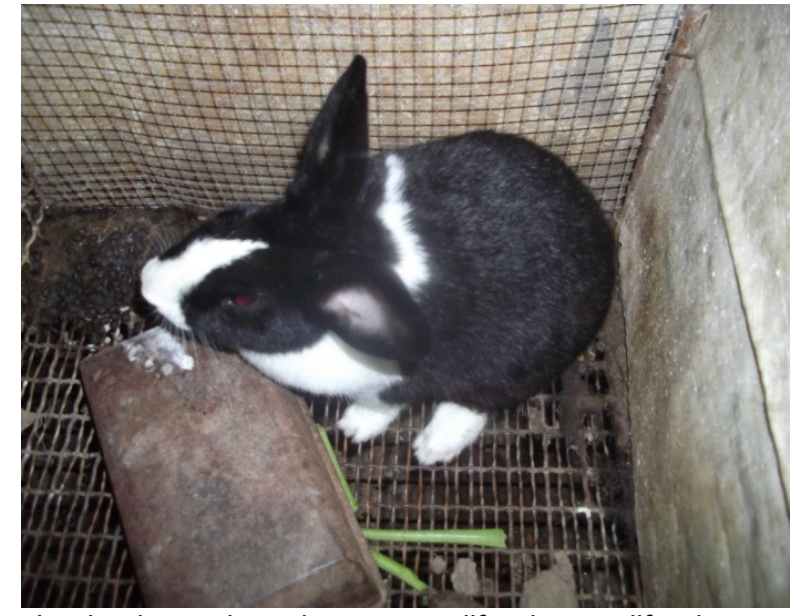

Lapine issue du croissement californien - californien

cardiovasculaires, des thromboses, d'athérosclérose et de cancer du colon (Appel et al., 2005; Bougnoux et Menanteau 2005 ). L'on note aussi dans la composition de cette viande des acides gras poly insaturés (acides linoléiques et alpha-linoléiques), dont leur consommation diminuerait l'incidence de l'hypertension humaine (Djoussé et al., 2005). L'objectif majeur poursuivi dans cette étude est d'appliquer les méthodes d'amélioration génétique aux lapins, par de divers croisements en décelant les produits plus performants (poids et taille) pouvant améliorer leur productivité pour l'avenir de l'élevage.

graminées et légumineuses, parmi lesquelles nous citons: Cynodon dactylon, Andropogon gayanus, Pennisetum purpureum, Tripsacum laxum, Imperata cylindrica, Eulesina Indica, Paspalum natatum.

Materiel : L'étude a porté sur un effectif total de 20 lapins adultes parmi lesquels 3 mâles de race Alaska, Néo-Zélandaise et Chinchilla, dont le poids varie entre 2,5 kg et 2,7 kg; et 17 femelles de race Argenté de Champagne (3), Californien (2), Chinchilla (3), NéoZélandais (6) et Papillon (3) ayant un score pondéral variant entre 2,4 et $3,8 \mathrm{~kg}$ de poids vif. Pour la récolte des données; hormis les animaux, nous avons utilisé une balance électronique de précision, un bloc note, un stylo, un marqueur ainsi que des fiches de pesée.

Methodes : Un plan factoriel de sélection de 6 races citées ci-haut, a été mis en place pour fournir les individus expérimentaux. Ces animaux issus d'un 
même troupeau ont été sélectionnés en fonction de leurs poids et performances. Ainsi 17 croisements ont été réalisés et ont permis l'obtention de 113 lapereaux. Nous avons procédé par la sélection des géniteurs mâles et femelles. Les reproductrices retenues, ont été celles qui ont connu deux mises bas chacune. Pour les mâles; une année d'âge au moins était le critère de sélection. Les animaux ont été nourris ad libitum avec de la verdure, carottes, feuilles des choux pommés, la patate douce, mais aussi d'un aliment concentré composé de $65 \%$ maïs, $25 \%$ soja, $2 \%$ sorgho sous forme de concassés, $1 \%$ sel, $3 \%$ minéraux et acides aminés (Kinophos, Premix), $4 \%$ Vitamines et coccidiostatiques (Amprolium). Ces animaux ont été alimentés selon le protocole établi et l'abreuvement y était assuré chaque matin, midi et soir. La répartition des mâles pour les femelles a été effectuée selon le poids et la race. Ainsi trois jours après la sélection, nous procédions à la mise au mâle de la femelle pour l'accouplement et observions l'effectivité de saillies pendant $1 / 4$ d'heure. Une fois les saillies terminées, la femelle était immédiatement retirée de la cage du mâle et remise dans celle de la maternité ; ensuite l'on notait la date de l'accouplement, le numéro de la femelle et celui du mâle. 5 jours plus tard, nous procédions aux saillies test c'est-à-dire, ramener la femelle dans la cage du mâle et observer pendant $1 / 4$ d'heure, si elle pouvait de nouveau accepter le mâle (test négatif). Lorsqu'elle était gestante, nous rédigions la date probable de la mise basse après saillies (28-32 jours). Après la mise basse, nous comptions à l'aide d'un bâton les petits lapereaux nés. Les lapereaux issus de ces mises bas étaient pesés aux jours : 15, 30, 45 (âge de sevrage) et 150 (entrée en reproduction) ; ensuite tous étaient élevés en cage collective grillagée avec un libre accès à l'eau et aliment. Compte tenu de nombreux facteurs qui, en post-partum et au sevrage plus particulièrement, paraissent influer sur les performances de reproduction; un protocole rigoureux basé sur la conduite d'élevage a été élaboré pour standardiser au maximum les conditions du milieu et l'état physiologique des géniteurs ainsi que des sevrés en post-sevrage. Enfin le calcul statistique a été effectué avec le logiciel Excel pour rechercher les moyennes et écart-type pour le gain pondéral ainsi que les gains quotidiens moyens des lapereaux aux jours 15,30 , au sevrage et à l'entrée en reproduction.

\section{RESULTATS}

Pour cette étude, les résultats sont repris dans les tableaux 1 à 6 .

Tableau 1 : Taille de la portée, nombre de petits sevrés et taux de mortalité dans différents croisements

\begin{tabular}{|c|c|c|c|}
\hline Croisement & Taille de la portée & Nb de petits sevrés & Mortalité (\%) \\
\hline $\mathrm{AL} X \mathrm{AR}$ & 8 & 7 & 0,88 \\
\hline $\mathrm{AL} X \mathrm{CA}$ & 6 & 6 & - \\
\hline AL X NZ & 9 & 9 & - \\
\hline AL X PA & 10 & 10 & - \\
\hline $\mathrm{AL} \times \mathrm{CH}$ & 9 & 0 & 7,96 \\
\hline $\mathrm{CH} \times \mathrm{CA}$ & 2 & 2 & - \\
\hline $\mathrm{CH} X \mathrm{NZ}$ & 9 & 4 & 4,42 \\
\hline $\mathrm{CH} \times \mathrm{PA}$ & 6 & 6 & - \\
\hline $\mathrm{CHXNZ}$ & 9 & 9 & - \\
\hline $\mathrm{CHXAR}$ & 4 & 4 & - \\
\hline $\mathrm{CHXNZ}$ & 6 & 6 & - \\
\hline $\mathrm{CHXCH}$ & 7 & 7 & - \\
\hline $\mathrm{CHX} \mathrm{NZ}$ & 6 & 4 & 1,77 \\
\hline NZ X PA & 6 & 6 & - \\
\hline $\mathrm{NZ} \times \mathrm{CH}$ & 5 & 5 & - \\
\hline $\mathrm{NZX}$ XR & 5 & 5 & - \\
\hline $\mathrm{NZX} \mathrm{NZ}$ & 6 & 6 & - \\
\hline Total & 113 & 96 & 15,04 \\
\hline Moyenne & $6,65 \pm 2,12$ & $84,90 \%$ & - \\
\hline
\end{tabular}




\section{Museba et al . J. Appl. Biosci. 2016 Contribution a l'étude des effets de croisement sur la taille de la portée et la croissance des lapereaux : Cas de la ferme agro- pastorale Jacarandas en R.D.Congo}

Ce tableau montre une moyenne de portée de 6,65 \pm 2,12 lapereaux. Ainsi la taille de la portée a été importante pour le croisement AL x PA (10 lapereaux), suivi de $\mathrm{AL} \times \mathrm{NZ}$ et $\mathrm{CH} \times \mathrm{NZ}$ (9 lapereaux). La plus faible portée a été observée chez $\mathrm{CH} \times \mathrm{CA}(2$ lapereaux). Concernant le taux de mortalité ; il a été de $15,04 \%$ sur l'ensemble des croisements effectués.
Cependant le taux de mortalité élevé a été observé respectivement pour les croisements $\mathrm{AL} \times \mathrm{CH}$ et $\mathrm{CH} x$ $\mathrm{NZ}$ en pré-sevrage. ce même tableau fait ressortir un nombre important des petits sevrés issus du croisement AL X PA, suivi respectivement de AL $X$ $\mathrm{NZ}$ et $\mathrm{CH} X \mathrm{NZ}$.

Tableau 2 : Poids moyen des lapereaux à 15 jours

\begin{tabular}{l|l|l}
\hline Croisement & Nb de petits & Poids moyen et écart-type (kg) \\
\hline AL X AR & 7 & $0,283 \pm 0,07$ \\
AL X CA & 6 & $0,244 \pm 0,03$ \\
AL X NZ & 9 & $0,226 \pm 0,04$ \\
AL X PA & 10 & $0,142 \pm 0,01$ \\
\hline CH X CA & 2 & $0,455 \pm 0,05$ \\
CH X NZ & 4 & $0,196 \pm 0,04$ \\
CH X PA & 6 & $0,327 \pm 0,05$ \\
CH X NZ & 9 & $0,296 \pm 0,04$ \\
CH X AR & 4 & $0,243 \pm 0,05$ \\
CH X NZ & 6 & $0,350 \pm 0,11$ \\
CH X CH & 7 & $0,342 \pm 0,08$ \\
CH X NZ & 4 & $0,241 \pm 0,01$ \\
\hline NZX PA & $0,248 \pm 0,04$ \\
NZX CH & 6 & $0,262 \pm 0,05$ \\
NZX AR & 5 & \\
NZ X NZ & 5 & $0,332 \pm 0,03$ \\
Moyenne & 6 & $0,621 \pm 0,12$ \\
\hline
\end{tabular}

Légende : AL: Alaska, AR: argenté, CH: chinchilla, CA: californien, NZ: néo-zélandais, PA: papillon, $\mathrm{Nb}$ : nombre

Le présent tableau montre un poids moyen des lapereaux de $0,300 \pm 0,11 \mathrm{~kg}$ à 15 jours d'âge. Le poids le plus élevé est observé pour le croisement NZ x
NZ $(0,621 \pm 0,12 \mathrm{~kg})$ et le plus faible pour AL $\times$ PA $(0,142 \pm 0,01 \mathrm{~kg}$

Tableau 3 : Poids moyen des lapereaux à 30 jours

\begin{tabular}{l|l|c|l}
\hline Croisement & Nb de petits & Poids moyen et écart-type $(\mathrm{kg})$ & \\
\hline & & $0,463 \pm 0,11$ \\
AL X AR & 6 & $0,611 \pm 0,05$ \\
AL X CA & 6 & $0,360 \pm 0,05$ \\
AL X NZ & 9 & $0,173 \pm 0,01$ \\
AL X PA & 10 & $0,721 \pm 0,17$ & \\
& 2 & $0,236 \pm 0,01$ \\
CH X CA & 4 & $0,604 \pm 0,08$ \\
CH X NZ & 6 & $0,487 \pm 0,07$ & \\
CH X PA & 9 & $0,495 \pm 0,12$ & \\
CH X NZ & 4 & $0,614 \pm 0,21$ \\
CH X AR & 6 & $0,485 \pm 0,09$ & \\
CH X NZ & 7 & $0,420 \pm 0,10$ & \\
CH X CH & 4 & $0,532 \pm 0,04$ & \\
CH X NZ & 6 &
\end{tabular}




\begin{tabular}{l|l|c|c}
\hline NZ X CH & 5 & $0,336 \pm 0,05$ & \\
NZ X AR & 5 & $0,431 \pm 0,06$ \\
NZ X NZ & 6 & $0,836 \pm 0,19$ & \\
& & $0,498 \pm 0,170$ & \\
\hline Moyenne & &
\end{tabular}

Légende : AL: Alaska, AR: argenté, $\mathrm{CH}$ : chinchilla, CA: californien, NZ: néo-zélandais, PA: papillon, $\mathrm{Nb}$ : nombre

De ce tableau se dégage une moyenne pondérale de $0,498 \pm 0,17 \mathrm{~kg}$. A cet âge, le produit issu de NZ x NZ a révélé un poids moyen élevé de $0,836 \pm 0,19$ $\mathrm{kg} / \mathrm{lapereau}$, suivi de $\mathrm{CH} \times \mathrm{CA}(0,721 \pm 0,17$ $\mathrm{kg} / \mathrm{lapereau})$. Le poids moyen le plus faible a été constaté chez AL x PA (de 0,173 $\pm 0,01 \mathrm{~kg} / \mathrm{lapereau}$ ).

Tableau 4 : Poids moyen des lapereaux à 45 jours

\begin{tabular}{l|l|l|l}
\hline Croisement & \multicolumn{2}{|l|}{ Nb de petits } & \multicolumn{2}{|c}{ Poids moyen et écart-type (kg) } \\
\hline & & & \\
AL X AR & 7 & $0,664 \pm 0,09$ & \\
AL X CA & 6 & $1,177 \pm 0,29$ & \\
AL X NZ & 9 & $0,667 \pm 0,08$ & \\
AL X PA & 10 & $0,545 \pm 0,07$ & \\
CH X CA & 2 & $1,004 \pm 0,24$ & \\
CHX NZ & 4 & $0,283 \pm 0,02$ & \\
CH X PA & 6 & $0,974 \pm 0,09$ & \\
CH X NZ & 9 & $0,738 \pm 0,05$ & \\
CH X AR & 4 & $0,889 \pm 0,13$ & \\
CH X NZ & 6 & $0,961 \pm 0,15$ & \\
CH X CH & 7 & $0,949 \pm 0,14$ & \\
CH X NZ & 4 & $0,628 \pm 0,06$ & \\
NZ X PA & 6 & $0,682 \pm 0,04$ & \\
NZ X CH & 5 & $0,503 \pm 0,05$ & \\
NZ X AR & 5 & $0,439 \pm 0,06$ & \\
NZ X NZ & 6 & $1,244 \pm 0,19$ & \\
\hline Moyenne & & $0,770 \pm 0,27$ &
\end{tabular}

Légende : AL: Alaska, AR: argenté, CH: chinchilla, CA: californien, NZ: néo-zélandais, PA: papillon, $\mathrm{Nb}$ : nombre

Le présent tableau fait ressortir un poids moyen de $0,770 \pm 0,27 \mathrm{~kg}$ à 45 jours d'âge. Le poids le plus élevé à cet âge a été observé pour le croisement NZ x NZ
$(1,244 \pm 0,19 \mathrm{~kg} /$ lapereau) et le plus faible, pour celui de $\mathrm{CH}$ x NZ (0,283 $\pm 0,02 \mathrm{~kg} / \mathrm{lapereau})$.

Tableau 5 : Poids moyen des lapins à 150 jours

\begin{tabular}{l|l|l|l}
\hline Croisement & Nb de petits & Poids moyen et écart-type (kg) & \\
\hline AL X AR & 7 & $1,703 \pm 0,20$ & \\
AL X CA & 6 & $2,183 \pm 0,30$ & \\
AL X NZ & 9 & $1,545 \pm 0,13$ & \\
AL X PA & 10 & $1,182 \pm 0,14$ & \\
CH X CA & 2 & $2,517 \pm 0,53$ & \\
CH X NZ & 4 & $1,816 \pm 0,07$ & \\
CH X PA & 6 & $2,482 \pm 0,20$ & \\
CH X NZ & 9 & $1,770 \pm 0,10$ & \\
CH X AR & 4 & $2,438 \pm 0,22$ & \\
CH X NZ & 6 & $2,434 \pm 0,40$ & \\
CHX CH & 7 & $2,447 \pm 0,20$ & \\
\hline
\end{tabular}




\begin{tabular}{|c|c|c|c|}
\hline \multicolumn{4}{|c|}{$\begin{array}{l}\text { Museba et al. J. Appl. Biosci. } 2016 \text { Contribution a l'étude des effets de croisement sur la taille de la } \\
\text { portée et la croissance des lapereaux : Cas de la ferme agro- pastorale Jacarandas en R.D.Congo }\end{array}$} \\
\hline \multicolumn{4}{|c|}{\begin{tabular}{|l|l|l|l}
$\mathrm{CH} X \mathrm{NZ}$ & 4 & $2,140 \pm 0,20$ & \\
\end{tabular}} \\
\hline \multicolumn{4}{|c|}{$\begin{array}{l}2,529 \pm 0,33 \\
2,090 \pm 0,40 \\
1,926 \pm 0,21 \\
2,900 \pm 0,40\end{array}$} \\
\hline Moyenne & & $2,130 \pm 0,45$ & \\
\hline
\end{tabular}

Légende : AL: Alaska, AR: argenté, $\mathrm{CH}$ : chinchilla, CA: californien, NZ: néo-zélandais, PA: papillon, Nb : nombre

À l'entrée en reproduction, la moyenne de poids de tous les lapins observés dans ce tableau est de 2,130 $\pm 0,45 \mathrm{~kg}$. Le poids moyen le plus élevé a été observé chez NZ x NZ (2,900 $\pm 0,40 \mathrm{~kg} / \mathrm{lapin})$ et le plus faible chez AL x PA (1,182 $\pm 0,14 \mathrm{~kg} / \mathrm{lapin})$.

Tableau 6 : Évolution du gain quotidien moyen (GQM) des lapereaux entre les jours 15-30, 30-45 et 45-150

\begin{tabular}{l|c|c|c|c}
\hline Croisement & Nb de lapereaux & $\begin{array}{c}\text { GQM au J 15-30 en } \\
(\mathbf{g})\end{array}$ & $\begin{array}{c}\text { GQM au J 30-45 en } \\
(\mathbf{g})\end{array}$ & $\begin{array}{c}\text { GQM au J 45-150 } \\
\text { en (g) }\end{array}$ \\
\hline AL X AR & 7 & 12,04 & 0,66 & 13,16 \\
AL X CA & 6 & 24,46 & 1,18 & 9,51 \\
AL X NZ & 9 & 8,92 & 0,67 & 13,32 \\
AL X PA & 10 & 2,12 & 0,55 & 13,04 \\
CH X CA & 2 & 17,70 & 1,00 & 15,14 \\
CH X NZ & 4 & 2,69 & 0,28 & 14,70 \\
CH X PA & 6 & 18,51 & 0,97 & 14,29 \\
CH X NZ & 9 & 12,74 & 0,73 & 14,23 \\
CH X AR & 4 & 16,83 & 0,89 & 14,25 \\
CH X NZ & 6 & 17,55 & 0,96 & 14,28 \\
CH X CH & 7 & 11,52 & 0,95 & 14,27 \\
CH X NZ & 4 & 12,97 & 0,63 & 14,22 \\
NZ X PA & 6 & 4,93 & 0,68 & 12,99 \\
NZ X CH & 5 & 6,61 & 0,50 & 15,11 \\
NZ X AR & 5 & 14,33 & 0,44 & 14,14 \\
NZ X NZ & 6 & & 1,24 & 15,01 \\
Total : & 96 & $12,11 \pm 6,2$ & $0,77 \pm 0,27$ & $13,85 \pm 1,35$ \\
\hline Moyenne & &
\end{tabular}

Légende : AL: Alaska, AR: argenté, $\mathrm{CH}$ : chinchilla, CA: californien, NZ: néo-zélandais, PA: papillon GQM : gain quotidien moyen, $\mathrm{j}$ : jour, $\mathrm{Nb}:$ nombre.

De ce tableau se dégagent différents GQM. Ainsi la moyenne de GQM la plus élevée a été observée aux j 45-150 (13,85 $\pm 1,35 \mathrm{~g} / \mathrm{j} / \mathrm{lapin})$ suivie de celle de GQM

\section{DISCUSSION}

Taille de la portée : Bien que le système d'élevage soit du type extensif et l'alimentation assurée plus par les légumes et autres herbages, et supplée par peu d'aliments concentrés; les investigations faites dans notre milieu de recherche ont révélé une moyenne de $6,65 \pm 2,12$ lapereaux à la première portée. Ces résultats semblent être rapprochés de ceux de Tudela aux j 15-30 (12,11 6 6,2 g/j/lapin). Celle de GQM aux j $30-45$ a été moins importante révélant à peine un gramme.

(1987), qui a trouvé dans une étude à Toulouse (France), une taille de la portée moyenne variant de $7,58 \pm 2,6$ à la première portée à $9,88 \pm 3,0$ lapereaux à la quatrième portée pour les lapines Néo-Zélandaises . Selon l'auteur, cela s'expliquerait par des variations du temps d'éclairement, de la température et la saison. De même que Ouyed et coll. (2007) ; qui ont donné des 
valeurs moyennes de taille de la portée variant entre 4 et 7 lapereaux pour les lapins élevés au Québec. Toutefois, certains auteurs estiment qu'en fonction du type génétique, l'accroissement de la production avec la taille de la portée cesse au-delà de 10 à 12 lapereaux allaités, voire moins pour les populations non sélectionnées (www.cuniculture.info/Docs/Biologie/ biologie-07-3.htm). Évolution pondérale des lapereaux : $\mathrm{Ne}$ pouvant pas manipuler les lapereaux à leur naissance, nos mesures de poids ont été faites à partir du quinzième jour de la naissance. Poids moyen des lapereaux à 15 jours : Selon Lebas (1991); Fielding et al. (1992) ; Heri (2010); le poids moyen à la naissance des lapereaux varie entre 50 et $90 \mathrm{~g}$. Ce poids évolue très rapidement de la naissance au sevrage, pendant que les lapereaux sont sous l'allaitement de leurs mères. Après quelques jours, ce poids quintuple; car durant trois semaines, les petits ne boivent que du lait. À partir de 18-20 jours ils diversifient peu à peu leur alimentation. Et pour les races lourdes, leur poids passe durant deux mois, de $50 \mathrm{~g}$ à $2500 \mathrm{~g}$ (poids moyen d'un lapin) (www.cuniculture.info/Docs/ indexbiol.htm). Le poids enregistré à la ferme Jacarandas montre une allure évolutive presque normale. II suffit de s'en rendre compte au tableau 2. Poids moyen des lapereaux à 30 jours : Vrillon et coll. (1979), ont révélé à 28 jours d'âge, des poids moyens des lapins issus des croisements CA x NZ et CA x CA, respectivement de $633 \mathrm{~g}$ et $562 \mathrm{~g}$. Heri (2010), dans une étude réalisée à la Cité des Jeunes à Lubumbashi (R.D.Congo), renseigne un poids moyen de $583 \mathrm{~g}$ à 30 jours d'âge. Quant aux travaux réalisés à Tizi-Ouzou en Algérie Par Lounaouci et coll. (2011), ont montré un poids moyen de $447 \mathrm{~g}$ au sevrage des lapereaux de différentes races réalisé à 28 jours de la naissance. Ce résultat est très peu différent de $455 \mathrm{~g}$ trouvé dans notre milieu d'étude à 30 jours d'âge. Poids moyen des lapereaux à 45 jours: Le poids moyen de 770

\section{CONCLUSION ET RECOMMANDATIONS}

II ressort de cette étude que le choix du lapin peut être déterminant pour l'élevage cunicole. Nous avons bien observé les effets du croisement dû au génotype du mâle sur la taille de la portée. Cependant, les portées nombreuses s'accompagnent des faibles poids des lapereaux qui influent sur leur croissance. Pour réussir l'élevage cunicole, il est impérieux de bien tenir des conditions d'exploitation telles que la conduite d'élevage, la gestion de la reproduction, l'alimentation et l'hygiène des locaux, la prophylaxie. Dans la présente étude, il apparaît que le mâle Alaska et Néo- g enregistré dans notre milieu d'étude à 45 jours de la naissance est très inférieur à celui prouvé par Gidenne et coll. (2012), dont le poids moyen de $1.583 \mathrm{~g}$ est obtenu entre 30 et 49 jours. La baisse de poids constatée dans notre étude s'expliquerait par la précarité des conditions d'exploitation (alimentation à base d'herbe supplée à peine de concentrés, prophylaxie, conditions environnementales non maîtrisées). Poids moyen des lapins adultes à 150 jours: Pour cet âge, plusieurs auteurs ont réalisé différents poids, notamment: Tudela (1987); qui a trouvé pour les lapins Néo-Zélandais à 4 mois d'âge, un poids moyen de $3.288 \mathrm{~g}$. Lebas et coll. (1996); Dans leur manuel, ont trouvé $3.200 \mathrm{~g}$ de poids moyen pour le lapin Néo-Zélandais blanc à 5 mois et demi d'âge. Pour Heri (2010) ; les poids variaient entre 2,5 et $3,7 \mathrm{~kg}$ à l'entrée en reproduction pour les 3 lapins adultes californien, Fauve de Bourgogne et Angora. Quant à Jaquet et coll. (2013), renseignent un poids moyen de $2.890 \mathrm{~g}$ pour les races moyennes à 4 mois d'âge. Le poids moyen obtenu dans notre milieu de recherche à 150 jours est de 2,130 $\pm 0,450 \mathrm{~kg}$. Ce poids est certes faible, compte tenu des races utilisées en croisement dans cet élevage ne sont pas pures, mais des produits qui ont subi un métissage et qui ont gardé certaines caractéristiques de leurs races d'origine. Gain Quotidien Moyen: Généralement dans la croissance du lapin, le GQM est très important en pré-sevrage qu'en post-sevrage. Romero et al. (2010) ; confirment que le GQM est excellent au début de la période d'avant sevrage, si les animaux sont soumis à une restriction alimentaire. Les différents GQM obtenus aux différents âges dans notre étude sont très faibles. Ce qui donne aussi un poids faible lors de l'admission des lapines à la reproduction $(2,130 \pm 0,450 \mathrm{~kg})$. Cette faible croissance reflète les conditions d'exploitation, mais aussi le grand métissage que ces animaux ont subi jusqu'à ce jour.

Zélandais sont d'une grande utilité pour l'augmentation de l'effectif de son cheptel; cependant leur utilisation devra être rationnelle. II ressort de cette étude que dans la limite des génotypes comparés, l'utilisation d'une race lourde en croisement permet d'augmenter la vitesse de croissance et l'efficacité alimentaire des lapins durant l'engraissement. II serait intéressant de vérifier si cette utilisation permet également l'amélioration des caractéristiques de la carcasse, à savoir le rendement et le poids des différentes parties après découpe. Une étude ultérieure est donc 
indispensable. L'utilisation des géniteurs à grande prolificité en croisement permet d'augmenter la productivité numérique des cheptels lapins. L'application d'une conduite de l'élevage adaptée

\section{BIBLIOGRAPHIE}

Akoutey et Kpodekon., 2009. Performances zootechniques des lapereaux recevant des alimentations granulés contenant «Pueraria phaseloid».

Appel LJ., Sacks FM., Carey VJ., Obarzanek E., Swain JF., Miller ER., Collin PR., Erlinger TP., Rosner BA., Laranjo NM., Charleston J., Mc Carron P., Bishop LM., 2005. Effects of protein mono unsaturated fat, and carbohydrate intake on blood pressure and serum lipids: Results of the omni Heart randomized trial. Jama Nov. 16 ; 294 (19): 2455-64.

Bougnoux P., Menanteau J., 2005. Dietary fatty acids and experimental carcinogenesis. Bull Cancer July, 92 (7): 685-96.

Combes S., 2004. Valeur nutritionnelle de la viande de lapin. INRA Productions Animales 17(5) :373383.

Combes S., Gidenne T., Boucher S., Fortum-Lamothe L., Bolet G., Coureau G., 2015. Lapereau de la naissance au sevrage quels outils pour les lapereaux plus robustes? France.

De Stefani E., Deneo-pellegrini H., 1999. Meat intake and risk of squamous cell esophageal cancer: a case- control study in Uruguay. Int. J. Cancer July 2; 82 (1): 33-7.

Djoussé L., Arnett DK., Pankou JS., Hopkins PN., Province MA., Ellison RC., 2005. Dietary linolenic acidis associated with a lower prevalence of hypertension in the NHLBI Family Heart study. March; 45 (3): 368-73.

Fao., 1998. Food Agriculture Organisation of the United Nations, Étude agricole de l'Organisation des Nations Unies pour I'Alimentation et l'Agriculture.

Fielding D., Donkey, Suhartanto B., Julliand V., Faurie F., 1992. Le technicien d'agriculture tropicale.

Gidenne T., Fortun-Lamothe L., Combes S., 2012. Restreindre l'ingestion du jeune lapin: des nouvelles stratégies pour renforcer sa santé digestive et améliorer son efficacité alimentaire, Toulouse .INRA Prod. Anim., 25 (4), 323-336.

Giovannucci E., Rimm EB., Stampfer MJ., Colditz GA., Anscherio A., Willett WC., 1994. Intake of fat, devrait permettre d'atteindre une augmentation de la vitesse de croissance avec un GQM satisfaisant pour l'éleveur.

meat and fiber in relation to risk of colon cancer in men. Cancer Res. May 1; 54 (9):2390-7.

Heri, 2010. Modèle de gestion de la cuniculture, UNILU, Faculté des sciences agronomiques, Lubumbashi.

Jaquet M., Bauwens V., Teller C., Dewasmes V., Maertens L., Marlier D., 2013. Contribution à la recherche des conditions optimales pour élever des lapins en parc hors-sol, Gembloux / Belgique.

Lebas, 1991. Alimentation pratique des lapins en engraissement (1ére partie). Cuniculture, 18, 273-281.

Lebas F., Lamboley B., Fortun-Lamothe L., 1996. Effects of dietary energy level and origin (starch vs oil) on rabbit milk gross and fatty acids composition. 6th world Rabbit Congress, Toulouse 9-12 july 1996, in (press).

Lounaouci, Ouyed G., Berchiche M., Gidenne T., 2011. Effet de l'incorporation de taux élevés (50\%) de son de blé dur sur la mortalité, la digestibilité, la croissance et la composition corporelle de lapins de la population blanche dans les conditions de productions algériennes. In Proc. 14ème journ. Rech. Cunicole Le Mans France 13-16.

Ouyed A., Lebas F., Lefrançois M., Rivest J., 2007. Performances de reproduction des lapines de races pures (Néo-zélandais blanc, Californien et Géant Blanc du Bouscat) et des croisés, en élevage assaini au Québec. 12émé journ. Rech. Cunicole, INRA-ITAVI. Le Mans 2728 Nov. 2007.

Piers LS., Walker KZ., Stoney RM., Soares MJ., O Dea K., 2003. Substitution of saturated with mono unsurated fat in a 4-week diet affects bady weight and composition of overweight and obese men. Br: J. Nutr. Sep; 90 (3): 717-27.

Riboli E., Norat T., 2001. Cancer prevention and diet: opportunities in Europe. Public health nutr. April; 4 (2B): 475-84.

Romero C., Cuesta S., Astillero J.R., Nicodemus N., De Blas C., 2010. Effect of early feed restriction on performance and health status in growing 
rabbits slaughtered at $2 \mathrm{~kg}$ live-weight. World Rabbit Sci., 18, 211-218.

Schiere J.B., 2004. Élevage des lapins dans les zones tropicales, 6ème Edition, Rome.

Tudela F., 1987. Effet de la taille de portée allaitée sur les performances de production des lapines. Rech. Cunicole Fr., Lyon 269-271.

Van, L., 1994. Étude de la géographie physique et humaine, Ed Loyola, Lubumbashi.
Vrillon J.L., Matheron G., De Rochambeau H., 1981. Réflexion à propos des études concernant le développement de l'élevage du lapin hors de l'hexagone. Cuniculture, 38, 121-123.

SITES INTERNET

www.cuniculture.info/Docs/Biologie/biologie-07-3.htm / Le 21 Sep -2015/ 11: 37 '.

www.cuniculture.info/Docs/indexbiol.htm / Le 21 Sep 2015/11:52'. 